\title{
CRITICAL GRAPHS FOR SUBPANCYCLICITY OF 3-CONNECTED CLAW-FREE GRAPHS
}

\author{
RONALD J. GOULD, TOMASZ LUCZAK, AND FLORIAN PFENDER
}

\begin{abstract}
Let $\mathcal{F}_{k}$ be the family of graphs $G$ such that all sufficiently large $k$-connected claw-free graphs which contain no induced copies of $G$ are subpancyclic. We show that for every $k \geq 3$ the family $\mathcal{F}_{k}$ is infinite and make the first step towards the complete characterization of the family $\mathcal{F}_{3}$.
\end{abstract}

\section{INTRODUCTION}

A graph is claw-free if it does not contain the complete bipartite graph $K_{1,3}$, the claw, as an induced subgraph. Claw-free graphs have been widely studied because of their special structural properties. In particular, the following type of problem has been considered by many authors:

Given a property $\mathcal{A}$ characterize the set $\overline{\mathcal{F}}_{k}(\mathcal{A})$ of all graphs $F$ such that each $k$-connected claw-free graph containing no induced copies of $F$ has $\mathcal{A}$.

Note that $\overline{\mathcal{F}}_{k}(A)$ can be equivalently defined as the set of graphs which appear as induced subgraphs in all $k$-connected claw-free graphs without property $\mathcal{A}$. Thus, if there exists at least one $k$-connected clawfree graph without property $\mathcal{A}$, then the family $\overline{\mathcal{F}}_{k}(A)$ is finite and is determined by a finite set of 'critical' graphs without $\mathcal{A}$. For instance, if $\mathcal{H}$ is the property that a graph is hamiltonian, then $\overline{\mathcal{F}}_{2}(\mathcal{H})$ consists of the path $P_{6}$, two other graphs (which we denote below by $F(1,1,1)$ and $F(0,1,2)$ respectively) and all their induced subgraphs (see [4] and references therein). Furthermore, $\overline{\mathcal{F}}_{2}(\mathcal{H})$ is determined by three critical graphs. At this moment we have only a partial characterization of the family $\overline{\mathcal{F}}_{3}(\mathcal{H}) \supseteq \overline{\mathcal{F}}_{2}(\mathcal{H})$ (see for instance [6]). Finally, it is believed each claw-free 4-connected graph is hamiltonian. If this is the case, then $\overline{\mathcal{F}}_{4}(\mathcal{H})$ contains all graphs.

Date: September 6, 2008.

1991 Mathematics Subject Classification. 05C38.

Key words and phrases. pancyclic graphs, claw-free graphs.

The second author partially supported by KBN grant 2 P03A 01623. 
Claw-free graphs used as 'critical' graphs are typically very small. On the other hand, if there exists $F$ such that there are only finitely many claw-free $k$-connected graphs without property $\mathcal{A}$ which contain no induced copy of $F$, then we might also say that prohibiting $F$, basically, forces $\mathcal{A}$. This is the case with a graph we denote below as $F(0,0,3)$; although it does not belong to $\overline{\mathcal{F}}_{2}(\mathcal{H})$ each 2-connected clawfree graph without an induced copy of $F(0,0,3)$ is hamiltonian provided it has at least ten vertices. Thus, instead of $\overline{\mathcal{F}}_{k}(\mathcal{A})$ one may want to look at the family $\mathcal{F}_{k}(\mathcal{A})$ which consists of all graphs $F$ for which there exists a constant $N_{F}$ such that each $k$-connected claw-free graph on at least $N_{F}$ vertices without an induced copy of $F$ has property $\mathcal{A}$. Note that, clearly, $\mathcal{F}_{k}(\mathcal{A}) \supseteq \overline{\mathcal{F}}_{k}(\mathcal{A})$. Moreover, in principle, at least for some properties $\mathcal{F}(\mathcal{A})$ might be an infinite proper subset of all finite graphs. In this paper we give an example of a property $\mathcal{A}$ for which this is indeed the case.

Let us observe first that $\mathcal{F}_{k}(\mathcal{H})$ is finite for $k=2$, 3. Indeed, let us take any graph $J$ which is obtained by attaching at least one pendant edge to each vertex of the Petersen graph. Let $G$ be the line graph of $J$. It is easy to check (see [6]) that $G$ is claw-free, 3-connected, and nonHamiltonian. Furthermore, although $G$ can be arbitrarily large, the number of its induced subgraphs which do not contain $K_{4}$ is bounded by an absolute constant. Since it is easy to see that no graph from $\mathcal{F}(\mathcal{H})$ contains $K_{4}$ (see Theorem 1 below), $\mathcal{F}_{3}(\mathcal{H})$ must be finite.

Thus, instead of $\mathcal{F}_{k}(\mathcal{H})$, we consider the family $\mathcal{F}_{k}=\mathcal{F}_{k}(\mathcal{S})$, where $\mathcal{S}$ is the property that a graph $G$ is subpancyclic, i.e., for every $\ell$, $3 \leq \ell \leq \operatorname{circ}(G), G$ contains a cycle of length $\ell$, where $\operatorname{circ}(G)$ denotes the length of the longest cycle in $G$.

Note first that since a graph is subpancyclic if each of its blocks is subpancyclic, $\mathcal{F}_{1}=\mathcal{F}_{2}$. The family $\mathcal{F}_{2}$ was studied by Faudree et al. [3] (see also Faudree and Gould [4]); we briefly recall their argument. Note that a cycle on $n \geq 4$ vertices is 2-connected but not subpancyclic, so the only possible candidates for members of $\mathcal{F}_{2}$ are paths. Now consider the graph $G_{n}$ which consists of the complete graph on the set $V_{1}$ of $2 n$ vertices, a perfect matching on the set $V_{2}$, where $V_{1} \cap V_{2}=\emptyset$ and $\left|V_{2}\right|=2 n$, and an additional perfect matching between these two sets. It is easy to see that $G_{n}$ is hamiltonian, but it contains no cycles on $4 n-1$ vertices. Moreover, $G_{n}$ contains no induced path $P_{7}$ on seven vertices and so $P_{k} \notin \mathcal{F}_{2}$ for $k \geq 7$. Finally, Faudree et al. [3] proved that each 2-connected claw-free graph on at least ten vertices which contains no induced copy of $P_{6}$ is subpancyclic. Hence, $\mathcal{F}_{2}=\left\{P_{3}, P_{4}, P_{5}, P_{6}\right\}$. 
In order to study the family $\mathcal{F}_{k}$ for $k \geq 3$ we introduce two types of graphs (see also Figure 1). By $E(r, s)$ we denote the graph which consists of two vertex-disjoint complete graphs on $r$ vertices which are connected by a path of length $s$ (it is a generalization of a graph $E$ introduced in [5]). By $F\left(t_{1}, \ldots, t_{r}\right)$, where $0 \leq t_{1} \leq \cdots \leq t_{r}$, we mean the graph which consists of the complete graph $K_{r}$ on $r$ vertices with paths of lengths $t_{1}, \ldots, t_{r}$ rooted at different vertices of $K_{r}$ (as mentioned above, graphs $F\left(t_{1}, t_{2}, t_{3}\right)$ emerge naturally in studying hamiltonicity of claw-free graphs). We also remark that in other papers, the graphs $E(3, s)$ and $F\left(t_{1}, t_{2}, t_{3}\right)$ are sometimes called double lasso and generalized net, respectively.
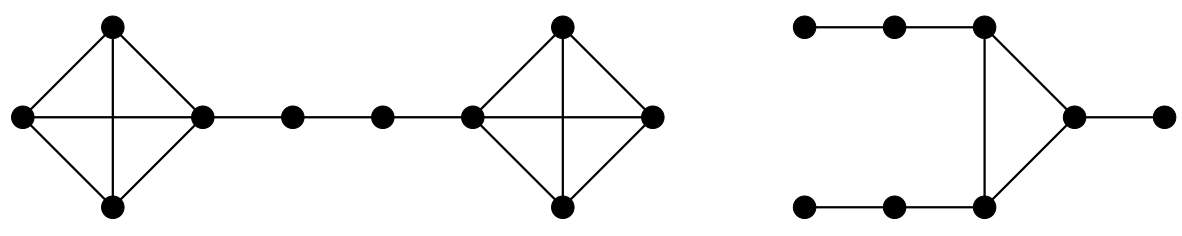

Figure 1. $E(4,3)$ and $F(1,2,2)$

Our first result narrows the set of possible candidates for elements of $\mathcal{F}_{k}$.

Theorem 1. Let $k \geq 3$ and let $r=r(k)=\lceil k / 2+1\rceil$. Then there exists $m=m(k)$ such that every graph $G \in \mathcal{F}_{k}$ is a subgraph of either $\mathrm{E}(r, 2 s+1)$ for some $s \geq 0$, or $F\left(t_{1}, t_{2}, \ldots, t_{r}\right)$ with $0 \leq t_{1} \leq \cdots \leq t_{r-2}$ and $t_{1} \leq m$.

Furthermore, $m(3)=2$, i.e., each graph from $\mathcal{F}_{3}$ is a subgraph of either $\mathrm{E}(r, 2 s+1)$ for some $s \geq 0$, or $F\left(t_{1}, t_{2}, t_{3}\right)$ with $0 \leq t_{1} \leq 2$.

We conjecture that, in fact, the above theorem gives an almost complete characterization of $\mathcal{F}_{k}$ and the following two conjectures hold.

Conjecture A. For every $k \geq 3$ there exists $\bar{m}(k)$ such that for each $0 \leq t_{1} \leq t_{2} \leq \cdots \leq t_{r}$, with $t_{r-2} \leq \bar{m}(k)$, there exists $\bar{N}$ such that each $k$-connected claw-free graph on at least $\bar{N}$ vertices without an induced copy of $F\left(t_{1}, \ldots, t_{r}\right)$ is subpancyclic.

Conjecture B. For every $k \geq 3$ and $s \geq 0$ there exists $\hat{N}$ such that each $k$-connected claw-free graph on at least $\hat{N}$ vertices without an induced copy of $\mathrm{E}(\lceil k / 2+1\rceil, 2 s+1)$ is subpancyclic.

In this paper we study more closely the family $\mathcal{F}_{3}$. It has been proved by the authors of this article (see [5]) that if a claw-free graph $G$ on at least eleven vertices is not pancyclic, then it contains induced 
copies of each of the graphs $P_{7}, £(3,1), F(0,0,4), F(0,1,3), F(1,1,2)$. Consequently, the above five graphs (and all their induced subgraphs) belong to $\mathcal{F}_{3}$. Here we show that Conjecture A holds with $\bar{m}(3)=2$, i.e., all graphs $F\left(t_{1}, t_{2}, t_{3}\right)$ with $t_{1} \leq 2$ belong to $\mathcal{F}_{3}$.

Theorem 2. The family $\mathcal{F}_{3}$ contains all graphs $F\left(t_{1}, t_{2}, t_{3}\right)$ with $t_{1} \leq 2$.

In particular, for $k \geq 3$ the family $\mathcal{F}_{k} \supseteq \mathcal{F}_{3}$ is infinite.

\section{Notation}

In this paper the term graph always stands for a simple graph. Graphs with multiple edges are called multigraphs. For all notation not defined here we refer the reader to [2]. We denote the vertex set and the edge set of a graph $G$ by $V(G)$ and $E(G)$ (or sometimes just $E)$, respectively. For a set of vertices $X \subseteq V(G), N(X)$ stands for the neighborhood of $X$, i.e., the set of all vertices outside $X$ adjacent to some vertices in $X$; sometimes we also use the closed neighborhood of $X$ defined as $N[X]=N(X) \cup X$. By $\langle X\rangle$ we mean the subgraph of $G$ induced by the set $X$. A vertex inside brackets denotes a vertex that may or may not be used in an induced subgraph.

For a directed path or cycle $H$ and two vertices $x, y \in V(H)$, we write $x H y$ for the $x-y$ path on $H$ following the direction of $H$. By $x^{--}, x^{-}$and $x^{+}, x^{++}$we denote the predecessors and successors of $x$ on $H$, respectively.

The distance between two vertices $x, y \in V(G)$ is the length of a shortest $x-y$ path, denoted by $d_{G}(x, y)$. If for any two vertices in a subgraph $H$ of $G, d_{H}(x, y)=d_{G}(x, y)$, then we say $H$ is distance preserving. The diameter of a connected graph, $\operatorname{diam}(G)$, is the maximum distance of two vertices in the graph. The girth of a graph is the length of a shortest cycle.

In Section 3 we give the proof to Theorem 1. In the following sections we first give a sketch of a fairly tedious proof of Theorem 2 (Section 4), and then proceed to prove it, first for graphs with small diameter (Section 5), and then for graphs with large diameter (Section 6).

\section{Proof of Theorem 1}

In this section we give a series of examples of $k$-connected claw-free graphs which are not subpancyclic. Clearly, each $H$ from $\mathcal{F}_{k}$ must appear as an induced subgraph in all but a finite number of graphs of the above kind, so in such a way we shall gradually narrow the family of possible candidates for graphs in $\mathcal{F}_{k}$.

For a multigraph $G$ we denote by $L(G)$ the line graph of $G$; the vertex set of $L(G)$ is the edge set of $G$, and two vertices in $L(G)$ are 
adjacent if the corresponding edges in $G$ share at least one vertex in $G$. The set of all multigraphs $F$ with $L(F)=G$ is $L^{-1}(G)$. The following fact list some useful properties of line graphs.

Fact 3. Let $G$ be a multigraph, let $L(G)$ be its line graph, and let $F$ be a graph. Then

(i) $L(G)$ is $k$-connected if and only if $G$ is essentially $k$-edgeconnected, i.e., the removal of fewer than $k$ edges in $G$ leads to a multigraph with exactly one non-trivial component,

(ii) $L(G)$ is claw-free,

(iii) $F$ is an induced subgraph of $L(G)$ if and only if $G$ contains one of the multigraphs in $L^{-1}(F)$ as a (not necessarily induced) submultigraph.

For a graph $G$, let $\operatorname{sub}(G)$ be the graph obtained from $G$ by a subdivision of each edge of $G$ into two edges.

We denote by $J(r, s)$ any $r$-regular essentially $(2 r-2)$-edge-connected graph with girth at least $s$. It is well known that for any numbers $r, s \geq 3$ such a graph $J(r, s)$ exists. In fact, a positive fraction of all $r$-regular graphs $(r \geq 3)$ have these properties, see for instance [1, Theorems II.19 and VII.32]. Observe that $\operatorname{sub}(J(r, s))$ is essentially $r$-edge-connected for $r \geq 2$.

Now, let $k \geq 3$ and $r=\lceil k / 2\rceil+1 \geq 3$. Then $L(J(r, r+2))$ is a $k$-connected claw-free graph which is not subpancyclic (it contains no cycle of length $r+1$ ), so no graph from $\mathcal{F}_{k}$ contains a complete graph on more than $r$ vertices. Furthermore, for every $s \geq(k+2) / 2$, the graph $L(\operatorname{sub}(J(k, s)))$ is a $k$-connected claw-free graph which contains cycles of length at least $2 s \geq k+2$ but no cycles of length $k+1$, and so it is not subpancyclic. Consequently, each graph in $\mathcal{F}_{k}$ is an induced subgraph of $L(T)$ for some $T$, where $T$ is a tree of maximum degree at most $r=\lceil k / 2\rceil+1$ in which each two vertices of degree larger than two are connected by a path of even length.

To narrow down the list of candidates further, consider the tree $T_{t}$, consisting of a path $v_{1} v_{2} \ldots v_{t+5}$ on $t+5$ vertices, and two vertices $u$ and $w$ with $u v_{3}, v_{t+4} w \in E(T)$. Then $L\left(T_{t}\right)$ is the graph obtained from $E(3, t)$ by adding a pendant edge to one of the two triangles. Moreover, $L^{-1}\left(L\left(T_{t}\right)\right)=\left\{T_{t}, T_{t}^{\prime}\right\}$, where $T_{t}^{\prime}$ is the multigraph obtained from $T_{t}$ through identification of $v_{t+5}$ and $w$ (see Figure 2). Our aim is to show that no graph containing $L\left(T_{t}\right)$ as an induced subgraph for some $t \geq 1$ can be member of $\mathcal{F}_{k}$.

To this end, start with the graph $J\left(k, k^{2}\right)$, and replace every edge by a path with $2 t+2$ edges with all but the middle two edges of this path being multiplied by $k-1$ (see Figure 3 for an example), and call the 


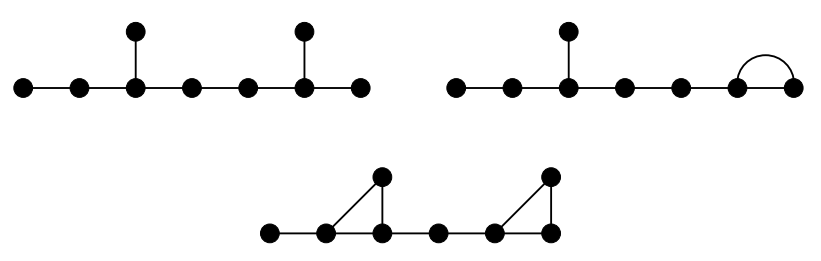

FiguRE 2. $T_{2}, T_{2}^{\prime}$ and $L\left(T_{2}\right)$

resulting multigraph $J_{t}\left(k, k^{2}\right)$. Then, $L\left(J_{t}\left(k, k^{2}\right)\right)$ is $k$-connected, and it contains no induced subgraph isomorphic to $L\left(T_{t}\right)$, since $J_{t}\left(k, k^{2}\right)$ does not contain $T_{t}$ or $T_{t}^{\prime}$ as a sub(multi)graph. All cycles in $L\left(J_{t}\left(k, k^{2}\right)\right)$ which correspond to cycles in the original $J\left(k, k^{2}\right)$ have length at least $k^{2}(2 t+2)>2 k^{2} t$. All other cycles in $L\left(J_{t}\left(k, k^{2}\right)\right)$ have length at most $k(t(k-1)+1) \leq k^{2} t$, so $L\left(J_{t}\left(k, k^{2}\right)\right)$ is not subpancyclic.
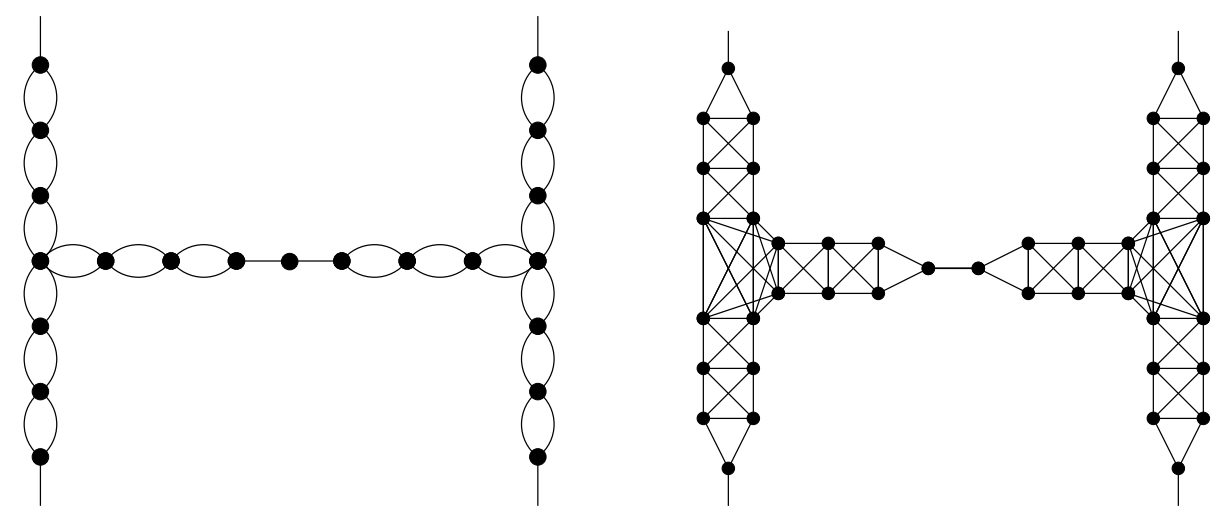

Figure 3. Parts of $J_{3}(3, s)$ and $L\left(J_{3}(3, s)\right)$

Thus, setting $r=\lceil k / 2\rceil+1$, all graphs from the family $\mathcal{F}_{k}$ are either subgraphs of $F\left(t_{1}, t_{2}, \ldots, t_{r}\right)$ or subgraphs of $E(r, t)$ for some odd $t$.

Finally, we bound $t_{i}$ for $i=0, \ldots, r-2$. In order to do that, take $n$ copies $G_{1}, \ldots, G_{n}$ of $J(k, k+1)$ and identify one vertex of $G_{i}$ with one vertex of $G_{i+1}$ for $i=1, \ldots, n-1$ in such a way that the graph $H_{k}^{n}$ obtained in this way has maximum degree $2 k$. Then the graph $L\left(\operatorname{sub}\left(H_{k}^{n}\right)\right)$ is claw-free and $k$-connected, but not subpancyclic (it contains no cycles of length $2 k+1)$. If $J(k, k+1)$ has $m$ vertices, then $L\left(\operatorname{sub}\left(H_{k}^{n}\right)\right)$ contains no copies of $F\left(t_{1}, t_{2}, t_{3}\right)$ with $t_{1} \geq 2 m$. This completes the proof of the main part of Theorem 1.

We conclude the proof with a slight refinement of the graph $H_{3}^{n}$ for the case $k=3$ to show that $m(3)=2$. Start with a cycle $C_{2 n}$ on $2 n$ vertices and double every other edge to get the multigraph $C_{2 n}^{\prime}$. 
Let $H_{3}^{n}=L\left(C_{2 n}^{\prime}\right)$. Then the graph $L\left(\operatorname{sub}\left(H_{3}^{n}\right)\right.$ ) (see Figure 4) is a hamiltonian claw-free 3-connected graph, which contains no cycles of length five. Furthermore, $L\left(s u b\left(H_{3}^{n}\right)\right)$ contains no induced copy of $F(3,3,3)$. Hence, the only possible candidates for the members of family $\mathcal{F}_{3}$ are paths, the graphs $E(3,2 s+1)$, where $s \geq 0$, and the graphs $F\left(t_{1}, t_{2}, t_{3}\right)$, where $t_{1} \leq 2$.

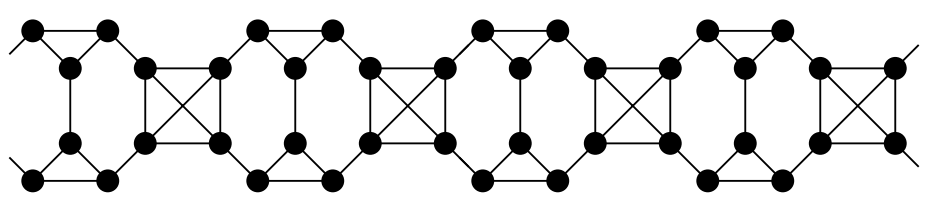

Figure 4. A part of $L\left(\operatorname{sub}\left(H_{3}^{n}\right)\right)$

\section{Proof Sketch For Theorem 2}

As the proof of Theorem 2 is rather tedious in parts, we want to give the reader a guideline with the main ideas and motivations before we get into the technical details.

We start with showing that all claw-free graphs with diameter much smaller than their order and minimum degree at least three are subpancyclic. The proof goes in two steps. In the first step, we use the fact that larger cycles must have shortcuts due to the small diameter, and thus we can find a slightly smaller cycle which we can then extend again to the desired length using the remaining vertices close to it. The second step is noting that such graphs have high maximum degree and therefore we can find cycles of small and moderate length in the closed neighborhood of a vertex.

In fact, we show a slightly stronger result in the first step. We show that for a given $\ell$-cycle $C$, we can find an $(\ell-1)$-cycle through a given edge of $C$ (Lemma 4). This stronger statement is used in Section 6 when we deal with graphs with large diameter.

If a graph $G$ has large diameter, then it contains a long distance preserving path $P$. Suppose that $G$ does not contain $F=F(r, k, k)$ as an induced subgraph for some fixed $r \ll k \ll \operatorname{diam}(G)$. Let $v$ be a vertex in $V(G) \backslash N[P]$, and suppose there is an induced path $R$ of length at least $r$ from $v$ to $N[P]$ with $N[P] \cap V(R)=\{w\}$, the final vertex of $R$. Then, $w$ is adjacent to one of the first or last $k$ vertices of $P$, as otherwise claw-freeness leads to a copy of $F$.

Therefore, $G$ falls in one of two classes. Either, every vertex in $V(G) \backslash N[P]$ has paths to at most one end section of $N[P]$, in which 
case we say that $G$ is linear, or there exists a vertex with paths into both end sections, in which case we call $G$ circular.

In the linear case, $G$ consists of a long mid section with all vertices within distance $r$ of $P$ and two end sections with diameter at most $2 k$. In the circular case, the structure is even simpler, as all vertices of $G$ are within distance $r$ of some long distance preserving cycle.

For the special case treated in Theorem 2, we have $r=2$. This gives us a lot of control over the mid section in the linear case, and over all of $G$ in the circular case. We take two disjoint long induced paths along a diameter path or a long distance preserving cycle, respectively, and show that there are so many edges between the two that we can find cycles of all lengths up to close to the order of the two paths.

If $G$ is circular, we then present a way to lengthen the distance preserving cycle one-by-one all the way up to $|V(G)|$, so in this case, $G$ is actually pancyclic. The argument in the linear case is somewhat more involved.

We first show that we can find cycles of all lengths up to the order of the mid section. But this still leaves the possibility of a (maximal) missing cycle length $\ell$ for a larger $\ell<\operatorname{circ}(G)$. If an $(\ell+1)$-cycle $C$ contains more than two thirds of the vertices in the mid section, then we can use our control there to find an $\ell$-cycle. Otherwise, $C$ must contain many vertices in one of the end sections. This is where Lemma 4 comes in again. After modifying $C$ a bit on the cut set separating the mid section from the end section, we can think of part of $C$ as one large cycle within the end section, one of its edges lying in the cut set. Now Lemma 4 lets us shorten this part of $C$ by one without affecting the connection to the remainder of $C$, finishing the proof.

\section{LARGE CLAW-FREE GRAPHS WITH SMALL DIAMETER}

In this section we show that all claw-free graphs with small diameter and minimum degree at least three are subpancyclic, provided they are large enough. The main tool for this is the following lemma which will be used again in Section 6 .

Lemma 4. Let $G$ be a connected claw-free graph with minimum degree at least three and diameter $d$. Suppose there exists an $(\ell+1)$-cycle $C$ in $G$ through an edge $x y \in E(C)$ for some $\ell \geq(24 d+22)^{d}$. Then $G$ also contains a cycle of length $\ell$ through $x y$.

Proof. Fix a direction on $C$ such that $y=x^{+}$. If, for any $v \in$ $V(C) \backslash\{x, y\}, v^{-}$and $v^{+}$are adjacent, then the assertion follows. Hence, we may assume for the remainder of the proof that no such short chord 
exists. This assumption implies that there is an edge $v^{-} w$ or $v^{+} w$ for every $w \in N(v) \backslash\left\{v^{-}, v^{+}\right\}$as otherwise $\left\langle v, v^{-}, v^{+}, w\right\rangle$ would be a claw.

The strategy of the proof goes as follows. First we find a cycle $C^{\prime}$ which is a little shorter than $C$ but which shares a long segments with $C$, and then use the above observation to lengthen $C^{\prime}$ to an $\ell$-cycle one vertex at a time. Let

$$
W:=\left\{v \in V\left(y^{+} C x^{-}\right): v \text { is not endvertex of a chord in } C\right\} .
$$

Since $G$ has minimum degree at least three, all vertices in $W$ have neighbors outside of $V(C)$. We consider two cases.

Case 1. Suppose $W=\emptyset$.

Let $y_{1} \in N(y) \cap V(C) \backslash\left\{y^{+}\right\}$such that $y$ has no neighbors in $y^{++} C y_{1}^{-}$ (possibly $y_{1}=x$ ). Let $u v$ be a chord of $C$ such that

- $u C v \subseteq y C y_{1}$,

- there are no other chords with both endvertices on $u C v$,

- $|V(y C u)|$ is minimal.

If $x^{-} y \in E$ and $N(x) \cap V\left(u^{+} C v^{-}\right) \backslash\left\{y^{+}\right\} \neq \emptyset$, let $x_{1} \in N(x) \cap$ $V\left(u^{+} C v^{-}\right) \backslash\left\{y^{+}\right\}$such that $\left|y C x_{1}\right|$ is minimal, and let $C^{\prime}=y x x_{1} C x^{-} y$. Otherwise, observe that $\left\langle\left\{x^{-}\right\} \cup\left(N(x) \cap V\left(u^{+} C v^{-}\right) \backslash\left\{y^{+}\right\}\right)\right\rangle$is complete to avoid a claw around $x$. Let $x_{1}=v$ and $C^{\prime}=x_{1} C u x_{1}$ in this case. In either case, $\left|V\left(C^{\prime}\right)\right| \leq \ell$, and $N(y) \cap\left(V(C) \backslash V\left(C^{\prime}\right)\right) \subseteq\left\{y^{+}\right\}$.

We shall insert all but one vertex of $V(C) \backslash V\left(C^{\prime}\right)$ back into $C^{\prime}$ to create the desired $\ell$-cycle. Let $w \in V(C) \backslash\left(V\left(C^{\prime}\right) \cup\left\{y^{+}\right\}\right)$. If $w \in N(x)$, we know by the observation above that $w x^{-} \in E$, and $w$ can be inserted between $x^{-}$and $x$. So assume that $w \notin N(x)$. Since $w \notin W$, there is a vertex $z \in V\left(x_{1}^{+} C x^{-}\right)$, with $w z \in E$. As above we may assume that $z^{-} z^{+} \notin E$. This implies that $w z^{-} \in E$ or $w z^{+} \in E$, otherwise there is a claw at $z$. This enables us to insert $w$ between $z^{-}$and $z$, or $z$ and $z^{+}$, respectively.

Inserting vertices of $V(C) \backslash\left(V\left(C^{\prime}\right) \cup\left\{y^{+}\right\}\right)$one by one yields the $\ell$-cycle. We will always use the first place $z z^{+} \in E\left(x_{1} C x\right)$ where the insertion is possible, (thus $w z^{-} \notin E$ ). The only problem we can encounter is a situation where we want to insert two different vertices $w, w^{\prime} \in V(C) \backslash\left(V\left(C^{\prime}\right) \cup\left\{y^{+}\right\}\right)$between the same vertices $z, z^{+} \in V\left(x_{1} C x\right)$. But then $w w^{\prime} \in E$ as otherwise $\left\langle z, z^{-}, w, w^{\prime}\right\rangle$ is a claw. Thus, $w$ and $w^{\prime}$ are neighbors on $C$ due to the choice of $u$ and $v$. In this case, we can extend $C^{\prime}$ through $z w w^{\prime} z^{+}$.

Case 2. Suppose $W \neq \emptyset$.

Since $W \neq \emptyset$, we have $V(C) \neq V(G)$. Thus, let $v \in V(G) \backslash V(C)$, and let $T^{\prime}$ be a spanning tree of $G$ such that $d_{T^{\prime}}(v, w)=d_{G}(v, w)$ 
for all $w \in V$, i.e., a breadth first search tree rooted at $v$. Let $T$ be the minimal subtree of $T^{\prime}$ with $V(T) \supseteq V(C) \cup\{v\}$. Direct $T$ away from $v$, and write $z_{-}$for the predecessor of a vertex $z \in V(T)$. By the diameter condition we know that $T$ has a vertex $z$ with at least $12 d+12$ outneighbors in $T$. Let $v_{1}, v_{2}, \ldots, v_{12 d+11} \in V(C) \backslash V(v T z)$ be $12 d+11$ vertices with $(V(C) \cup V(v T z)) \cap V\left(z T v_{i}\right) \subseteq\left\{z, v_{i}\right\}$. Out of these vertices we pick seven vertices $y_{1}, y_{2}, \ldots, y_{7}$ in this order on $y^{++} C x^{--}$, such that $\left|V\left(y_{i} C y_{i+1}\right)\right| \geq 2 d+2$ for $1 \leq i \leq 6$. By the pigeon hole principle it is true that $\left|V\left(y_{i}^{+} C y_{i+1}^{-}\right) \cap W\right| \leq \frac{|W|}{6}$ for some $1 \leq i \leq 6$. As it does not affect the remainder of the proof, we may assume that $i=1$.

If possible, choose $u, w \in V\left(y_{1} C y_{2}\right)$, such that $u w$ is a chord of $C$, and no other chords are inside $u C w$, and let $C^{\prime}=w C u w$. If there is no such chord, let $u=y_{1}, w=y_{2}$ and $C^{\prime}=w C u T w$. The only way that $C^{\prime}$ is not a cycle is that $y_{1} T y_{2}$ uses $z$ and $z \in V(C)$ (and thus $z \neq v)$. In this case, let $z_{1}, z_{2}$ be the neighbors of $z$ on $y_{1} T y_{2}$. None of the edges $z_{-} z_{1}, z_{-} z_{2}$ can exist by the distance property of $T$. Hence, $z_{1} z_{2} \in E$ to avoid a claw at $z$. Now choose $C^{\prime}=w C u T z_{1} z_{2} T w$. In any case, $\left|V\left(C^{\prime}\right)\right| \leq \ell$.

Let $X=\left\{z \in V\left(u^{+} C w^{-}\right) \backslash W: N(z) \cap V\left(w^{+} C u^{-}\right) \subseteq\{x, y\}\right\}$, let $X_{1}=X \cap N(x), X_{2}=X \cap N(y)$. These are vertices which can create some problems later, and we show here that there are very few of them. Note that $\left\langle X_{1}\right\rangle$ is complete, otherwise there is a claw centered around $x$, using $x^{-}$and two independent vertices in $X_{1}$. Similarly, $\left\langle X_{2}\right\rangle$ is complete. This implies that $\left|X_{1}\right|,\left|X_{2}\right| \leq 2$ as there are no chords inside $u^{+} C w^{-}$. If $|X|>2$, there are vertices $x_{1} \in X_{1}$ and $x_{2} \in X_{2}$ with $x_{1} x_{2} \notin E$. To avoid claws around $x$ and $y$, we must have $x^{-} y, x y^{+} \in E$ and thus $x^{-} y^{+} \in E$. In this case we choose a cycle $C^{\prime \prime}=$ $y^{+} C x_{2} y x x_{1} C x^{-} y^{+}$or $C^{\prime \prime}=y^{+} C x_{1} x y x_{2} C x^{-} y^{+}$, such that $\left|V\left(C^{\prime \prime}\right)\right| \leq \ell$. Otherwise, let $C^{\prime \prime}=C^{\prime}$. In either case, $\left|X \cap\left(V(C) \backslash V\left(C^{\prime \prime}\right)\right)\right| \leq 2$.

Just as in Case 1, we can now insert vertices from $V(C) \backslash\left(V\left(C^{\prime \prime}\right) \cup\right.$ $X \cup W)$ into $C^{\prime \prime}$ to get a longest possible cycle $C^{\prime \prime \prime}$ with

$$
m-2-\left\lfloor\frac{|W|}{6}\right\rfloor \leq\left|V\left(C^{\prime \prime \prime}\right)\right| \leq m-1 .
$$

Note that all these insertions happen at sections of $C$ where $C^{\prime \prime}$ and $C$ are identical. No vertex is inserted next to $x$ or $y$ as vertices of $X$ are not inserted, and no vertex is inserted next to a vertex of $W$, since these vertices are not endvertices of chords. Note also that, since the minimum degree of $G$ is at least three, each vertex in $W \cap V\left(C^{\prime \prime \prime}\right)$ has at least one neighbor outside $V\left(C^{\prime \prime \prime}\right)$. Furthermore, each such neighbor can be adjacent to at most four vertices from $W$ without creating a 
claw. Therefore,

$$
\left|N(W) \cap V\left(C^{\prime \prime \prime}\right)\right| \geq\left\lceil\frac{1}{4} \times \frac{5}{6}|W|\right\rceil \geq\left\lfloor\frac{|W|}{6}\right\rfloor+1 .
$$

Since $G$ is claw-free, all these vertices can be inserted into $C^{\prime \prime \prime}$ one by one (if they are not already part of $C^{\prime \prime \prime}$ as vertices of $u T w$ ), and so $G$ contains an $\ell$-cycle, contrary to our assumption.

As a consequence of Lemma 4, we get the following theorem.

Theorem 5. For every $d$ there exists an $n=n(d)$ such that each connected claw-free graph $G$ with at least $n$ vertices, diameter at most d, and minimum degree at least three is subpancyclic.

Proof. Let $G$ be a connected claw-free graph with $n$ vertices, diameter at most $d$, and minimum degree at least three. Lemma 4 implies that $G$ contains a cycle of length $\ell$, for each $\ell$ such that $\operatorname{circ}(G) \geq \ell \geq$ $\ell_{0}=(24 d+22)^{d}$. Note also that from the fact that $G$ has diameter at most $d$ it follows that it contains a vertex $v$ of degree $D \geq(n-1)^{1 / d}$.

Since $G$ is claw-free, $\langle N(v)\rangle$ has independence number at most two, and it is an easy observation that $\langle N(v)\rangle$ contains a path of length $\lceil D / 2\rceil$. Then $\langle N[v]\rangle$ contains a cycle of length $\ell$ for each $\ell, 3 \leq \ell \leq$ $D / 2+1$, so it is enough to choose $n$ large enough to have $(n-1)^{1 / d} / 2 \geq$ $\ell_{0}-2$.

\section{Graphs with Large Diameter}

For the proof of Theorem 2 it suffices to show that $\mathcal{F}_{3}$ contains $F(2, k, k)$ for all $k \geq 10$. So let $k \geq 10$ and $F=F(2, k, k)$.

We start with the following simple lemma.

Lemma 6. For $u, v \in V(G)$, let $P$ be an induced $u-v$ path in a clawfree graph $G$, and let $X \subseteq N\left(u^{+} P v^{-}\right)$. Then $G$ contains a $u-v$ path $R$ with $V(R)=V(P) \cup X$ on which all vertices of $V(P)$ appear in the same order as on $P$.

Proof. Without loss of generality we may assume that $V(G)=X \cup$ $V(P)$. For $w \in V(P) \backslash\{u, v\}$, let $X_{w}=X \cap\left(N(w) \backslash N\left(w^{-}\right)\right)$. Since $G$ is claw-free, every $\left\langle X_{w} \cup\left\{w^{+}\right\}\right\rangle$is complete.

Suppose first that $\langle N(u)\rangle$ is 2-connected. Since $G$ is claw-free, $\langle N(u)\rangle$ contains no independent sets of size three and thus it is hamiltonian. Consequently, we can construct a path $Q$ from $u$ to $u^{++}$through all vertices of $N[u]$. If $\langle N(u)\rangle$ is not 2-connected, then $\langle N(u)\rangle$ consists of two complete graphs sharing at most one vertex, where one of them contains $u$, the other $u^{++}$. Then, again, we can construct 
a path $Q$ from $u$ to $u^{++}$through all vertices of $N[u]$. Now let $R=$ $u Q u^{++} X_{u^{++}} u^{3+} \ldots v^{-} X_{v^{-}} v$.

The next lemma tells us a lot about the structure of $G$.

Lemma 7. Let $G$ be $\left\{K_{1,3}, F\right\}$-free, and let $P=v_{0} v_{1} \ldots v_{4 k+3}$ be an induced path in $G$. Let $x \in N\left(v_{2 k+1}\right) \cap N\left(v_{2 k+2}\right), u \in N(x) \backslash N[P]$, and let $w \in N[u] \backslash N[P]$. Then $N[w]=N[u]$.

Proof. First note that $w x \in E$ since otherwise $V(P) \cup\{x, u, w\}$ contains $F$. Suppose that $N(u) \neq N(w)$. Because of symmetry, we may assume that there exists $z \in N(u) \backslash N(w)$. If $z \in N\left(v_{k+1} P v_{3 k+2}\right)$, then $\langle V(P) \cup$ $\{z, u, w\}\rangle$ contains $F$, a contradiction. Hence $z \notin N\left(v_{k+1} P v_{3 k+2}\right)$ and $x z \notin E$ to avoid a claw $\left\langle x ; v_{2 k+1}, w, z\right\rangle$. But now $\left\langle V\left(v_{k+1} P v_{3 k+2}\right) \cup\right.$ $\{x, u, z\}\rangle$ is a copy of $F$. This contradiction completes the proof of the lemma.

We call $G$ circular if it contains a distance preserving cycle of length greater than $10 k$ as a subgraph, and linear otherwise. We get the following two corollaries from Lemma 7.

Corollary 8. If $G$ is $\left\{K_{1,3}, F\right\}$-free and contains an induced cycle $C$ with $|V(C)| \geq 4 k+5$, then $V(G)=N[N[C]]$. Furthermore, the diameter of $G$ is at most $\frac{|V(C)|}{2}+3$.

Corollary 9. If $G$ is $\left\{K_{1,3}, F\right\}$-free and linear with diameter $N>$ $10 k$, and $P=v_{0} v_{1} \ldots v_{N}$ is a diameter path of $G$, then every vertex $v \in V(G) \backslash N[N[P]]$ has distance at most $2 k+1$ from one of $v_{2 k}$ and $v_{N-2 k}$.

We are ready to prove the existence of short and medium length cycles in the linear case now.

Lemma 10. Suppose that $G$ is 3-connected, $\left\{K_{1,3}, F\right\}$-free and linear, $N>10 k$, and that $P=v_{0} v_{1} \ldots v_{N}$ is a distance preserving path in $G$. Then $G$ contains cycles of all lengths $\ell$ for $3 \leq \ell \leq 2 N-16 k$.

Proof. Let $C$ be a minimal cycle through $v_{0}$ and $v_{N}$. Then $v_{0} C v_{N}$ and $v_{N} C v_{0}$ are induced paths, and we can apply Lemma 7 to them. Note also that all chords of $C$ have ends in both $v_{0}^{+} C v_{N}^{-}$and $v_{N}^{+} C v_{0}^{-}$. Let $x_{1} y_{1}$ and $x_{2} y_{2}$ be chords of $C$ such that

- $\left|V\left(v_{0} C x_{1}\right)\right|,\left|V\left(y_{1} C v_{0}\right)\right|,\left|V\left(x_{2} C v_{N}\right)\right|,\left|V\left(v_{N} C y_{2}\right)\right|>2 k$, and

- $\left|V\left(y_{1} C x_{1}\right)\right|$ and $\left|V\left(x_{2} C y_{2}\right)\right|$ are minimal under this condition.

Such chords exist due to Corollary 8 and the fact that $v_{0}$ and $v_{N}$ have distance $N$. Furthermore, by the same reasons we have $\left|V\left(x_{1} C x_{2} y_{2} C x_{2} x_{1}\right)\right| \geq$ 
$2 N-16 k$. Let

$$
W=\left(V\left(x_{1} C x_{2}\right) \backslash N\left(v_{N} C v_{0}\right)\right) \cup\left(V\left(y_{2} C x_{2}\right) \backslash N\left(v_{0} C v_{N}\right)\right) .
$$

Then $W$ is an independent set by Lemma 7 applied to $v_{0} C v_{N}$ and $v_{N} C v_{0}$, since any two consecutive vertices on each of the two paths have different closed neighborhoods.

Claim 1. If $x_{3} y_{3}$ and $x_{4} y_{4}$ are chords in $C$, where $v_{0}, x_{3}, x_{4}, v_{N}, y_{3}, y_{4}$ appear on $C$ in this order, then $\left\langle x_{3}, x_{4}, y_{3}, y_{4}\right\rangle$ is a complete graph.

First, $x_{4}=x_{3}^{+}$and $y_{4}=y_{3}^{+}$as otherwise we could find a shorter cycle through $v_{0}$ and $v_{N}$. And then, $x_{3} y_{4}, x_{4} y_{3} \in E$ to avoid claws. $\diamond$

Claim 2. If $x y$ is a chord in $C$, and $x^{-} y, x y^{+} \notin E$, then $\left\langle x, x^{+}, y, y^{-}\right\rangle$ is a complete graph.

We have $x y^{-}, x^{+} y \in E$ to avoid claws, the existence of the remaining edge follows from Claim 1.

Suppose that $\left\langle V\left(x_{1} C x_{2} y_{2} C y_{1} x_{1}\right)\right\rangle$ contains an $(\ell+1)$-cycle $C=$ $x_{3} C x_{4} y_{4} C y_{3} x_{3}$ using exactly two chords of $C$, but no $\ell$-cycle with this property for some $\ell$ with $3 \leq \ell \leq 2 N-15$. Then $\left\langle x_{3}^{-}, x_{3}, y_{3}, y_{3}^{+}\right\rangle$ and $\left\langle x_{4}, x_{4}^{+}, y_{4}^{-}, y_{4}\right\rangle$ are complete by Claim 2 . If any of the edges $x_{3}^{+} y_{3}^{-}, x_{3}^{+} y_{3}^{--}, x_{3}^{++} y_{3}^{-}, x_{4}^{-} y_{4}^{+}, x_{4}^{-} y_{4}^{++}, x_{4}^{--} y_{4}^{+}$are present, we can find an $\ell$-cycle using only two chords of $C$. If this cycle contains vertices in $\left\{x_{3}^{-}, y_{3}^{+}, x_{4}^{+}, y_{4}^{-}\right\}$, we can subsequently skip these vertices one-by-one to create shorter cycles, ending up with an $(\ell-1)$-cycle or an $(\ell-2)$-cycle using only two chords of $C$ with all vertices inside $V\left(x_{1} C x_{2} y_{2} C y_{1} x_{1}\right)$.

If none of these edges exist, $x_{3}^{+}, y_{3}^{-}, x_{4}^{-}, y_{4}^{+} \in W$ and $x_{3}^{++} y_{3}^{--}, x_{4}^{--} y_{4}^{++} \in$ $E$ by Claim 1 . Now $x_{3}^{-} C x_{4}^{--} y_{4}^{++} C y_{3}^{+} x_{3}^{-}$is an $(\ell-1)$-cycle using only two chords of $C$ (which can again be reduced to find a cycle with all vertices inside $\left.V\left(x_{1} C x_{2} y_{2} C y_{1} x_{1}\right)\right)$. If $\ell>5$, we can extend this cycle through a neighbor of $x_{3}^{+}$to construct an $\ell$-cycle.

Finally, if $\ell=5$, we have $x_{3}^{+}=x_{4}^{-}$and $y_{3}^{-}=y_{4}^{+}$. Let $x \in\left(N\left(x_{3}^{+}\right) \cap\right.$ $\left.N\left(v_{N} C v_{0}\right)\right) \backslash\left\{x_{3}, x_{4}\right\}$. This vertex exists by Lemma 7 since $G$ is 3 connected. If $x \in N\left(y_{4} C y_{3}\right)$, then we can find a 5-cycle through $x$. Otherwise we can reduce $|V(C)|$, a contradiction.

Now we show Theorem 2 in the case of circular $G$.

Lemma 11. Suppose that $G$ is 3-connected, $\left\{K_{1,3}, F\right\}$-free and $G$ contains a distance preserving cycle of length $N>20 k$. Then $G$ is pancyclic.

Proof. For cycle lengths $\ell$ with $3 \leq \ell<N$, we use a strategy very similar to the proof of Lemma 10. Let $C=v_{0} v_{1} \ldots v_{N-1} v_{0}$ be a distance 
preserving cycle of length $N$ in $G$. Treat all indices in the following modulo $N$.

Let $u \in V(G) \backslash V(C)$. Since $C$ is distance preserving, we have $N(N(u)) \cap V(C) \subseteq V\left(v_{i} C v_{i+4}\right)$ for some $0 \leq i \leq N-1$. This, together with Lemma 7 , guarantees that $N\left[N\left[v_{i} C v_{i+3}\right]\right]$ is a cut set of $N\left[N\left[v_{i-k} C v_{i+k}\right]\right]$ separating $v_{i-k}$ and $v_{i+k}$ for all $i$.

Consider $G_{i}=G \backslash N\left[N\left[v_{i} C v_{i+3}\right]\right]$. If $G_{i}$ contains a cycle through $v_{i-k}$ and $v_{i+k}$, we can use a shortest such cycle in place of $C$ in the proof of Lemma 10, and show that $G_{i}$ contains cycles of all lengths $\ell$ for $3 \leq \ell \leq 2(N-2 k)-16 k$.

Otherwise, there is a $j$ with $|i-j|>k$ such that $v_{j}$ is a cut vertex of $G_{i}$ separating $v_{i-k}$ and $v_{i+k}$. Repeat the argument to show that $G_{j}$ contains cycles of all lengths $\ell$ for $3 \leq \ell \leq 2(N-2 k)-16 k$ unless there is a $j^{\prime}$ with $\left|j^{\prime}-j\right|>k$ such that $v_{j^{\prime}}$ is a cut vertex of $G_{j}$ separating $v_{j-k}$ and $v_{j+k}$. But then $\left\{v_{j}, v_{j^{\prime}}\right\}$ is a cut set of $G$, contradicting that $G$ is 3 -connected.

For $\ell \geq N$, we start with $C$ and extend it one vertex at a time. By Lemma 6 , we can include any set $X \subseteq N(C)$ into the cycle.

For vertices $u \in N(N[C])$, let $Z_{u}=\{w \in N(N[C]): N[u]=N[w]\}$. If $\left|N(u) \cap N\left(v_{i}\right) \cap N\left(v_{i+1}\right)\right| \geq 2$ for any $i$, we can extend the cycle through these two vertices in $N(C)$ and any number of vertices in $Z_{u}$. Otherwise, $u$ has neighbors in both $N\left(v_{i}\right) \cap N\left(v_{i+1}\right)$ and $N\left(v_{i}\right) \cap N\left(v_{i-1}\right)$ for some $i$ since $G$ is 3-connected. Again, we can extend the cycle through these two neighbors in $N(C)$ and any number of vertices in $Z_{u}$. If there are two (or more) vertices $u_{1}, u_{2}$ with $N\left[u_{1}\right] \neq N\left[u_{2}\right]$ and $\left|N\left(u_{1}\right) \cap N\left(v_{i}\right) \cap N\left(v_{i+1}\right)\right|=\left|N\left(u_{2}\right) \cap N\left(v_{i}\right) \cap N\left(v_{i+1}\right)\right|=\mid N\left(u_{1}\right) \cap N\left(u_{i}\right) \cap$ $N\left(v_{i-1}\right)|=| N\left(u_{2}\right) \cap N\left(v_{i}\right) \cap N\left(v_{i-1}\right) \mid=1$, we can go back and forth through any number of vertices in $Z_{u_{1}}$ and $Z_{u_{2}}$. Note that Lemma 7 and claw-freeness of $G$ guarantee that $u_{1}$ and $u_{2}$ have no common neighbors, so we can perform all these insertions independently.

Because of Lemmas 10 and 11 for the proof of Theorem 2 it remains to show the following lemma.

Lemma 12. Suppose that $G$ is 3-connected, $\left\{K_{1,3}, F\right\}$-free and linear with diameter $N>10 k$. For every $\ell \geq 2 N-16 k$, if $G$ contains an $\ell$-cycle, then $G$ contains an $(\ell-1)$-cycle.

Proof. In the following two claims, we show that a lot of the sets $N\left[v_{i}\right] \cap$ $N\left[v_{i+1}\right]$ are cut sets of $G$.

Claim 3. Suppose there exists an $u \in N(N[P])$ such that $N(N(u)) \cap$ $V(P) \subseteq V\left(v_{i} P v_{j}\right), v_{i}, v_{j} \in N(N(u))$ and $2 k+1 \leq i \leq j \leq N-2 k-1$. 
Then $N\left[v_{i}\right] \cap N\left[v_{i+1}\right]$ and $N\left[v_{j-1}\right] \cap N\left[v_{j}\right]$ are cut sets of $G$ separating $v_{0}$ and $v_{N}$.

By symmetry, it is enough to prove the statement for the first set. For the sake of contradiction, assume that for $X=N\left[v_{i}\right] \cap N\left[v_{i+1}\right]$ there is a path from $v_{0}$ to $v_{N}$ in $G-X$.

Let $R$ be such a path which contains the minimal number of vertices outside of $P$. It is easy to see that $R$ contains exactly two vertices $y_{1}$ and $y_{2}$ (in this order) in $N(P)$, and at most one vertex $w$ outside of $N[P]$.

Let $x \in N\left(v_{i}\right) \cap N(u)$. The following edges can not exist (see also Figure 5): $u y_{1}$ (since $v_{i-1} \notin N(N(u))$ ), uw (by Lemma 7 with $u y_{1} \notin E$ ), $u y_{2}$ (claw centered at $\left.y_{2}\right), v_{i} y_{2}\left(y_{2} \notin X\right), w x$ (claw centered at $\left.x\right), x_{1} y_{2}$ (claw centered at $y_{2}$ ), and $x y_{1}$ (claw centered at $y_{1}$ ). Furthermore, one of $v_{i} y_{1}$ and $v_{i-2} y_{1}$ is not an edge, otherwise there is a claw centered at $y_{1}$.

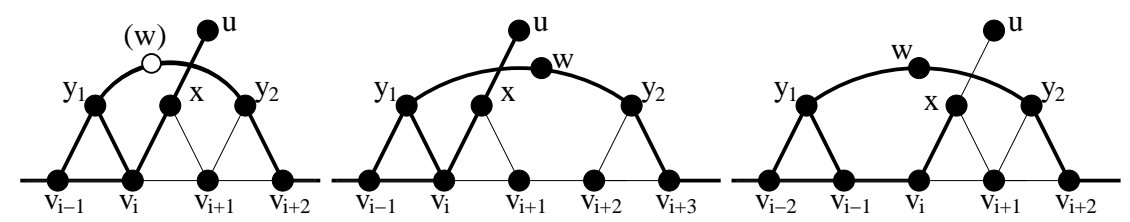

Figure 5. The three possible configurations in the proof of Claim 3

But now it is easy to extend $R$ to a copy of $F$ through $v_{i}, x$ and one of $v_{i-1}$ and $u$, a contradiction.

Claim 4. For $2 k+5 \leq i \leq N-2 k-8$, at least one of the sets $N\left[v_{i}\right] \cap N\left[v_{i+1}\right], N\left[v_{i+1}\right] \cap N\left[v_{i+2}\right]$ and $N\left[v_{i+2}\right] \cap N\left[v_{i+3}\right]$ is a cut set of $G$ separating $v_{0}$ and $v_{N}$.

Suppose that none of the three sets cuts $G$. By Claim 3, no vertex in $N(N[P])$ has distance 2 to $v_{i} P v_{i+3}$. Thus, there exist three edges $x_{i-1} x_{i+1}, x_{i} x_{i+2}$ and $x_{i+1}^{\prime} x_{i+3}$ with $x_{j}, x_{j}^{\prime} \in N\left(v_{j}\right) \cap N\left(v_{j+1}\right)$ for $i-1 \leq$ $j, j^{\prime} \leq i+3$ (see Figure 6 ). The edges $x_{i-1} x_{i}$ and $x_{i+2} x_{i+3}$ would lead to claws at $x_{i-1}$ and $x_{i+3}$, respectively, and similarly, any edge of $x_{i} x_{i+1}, x_{i} x_{i+1}^{\prime}, x_{i+1} x_{i+2}$ and $x_{i+1}^{\prime} x_{i+2}$ would lead to a claw at $x_{i+1}$ or $x_{i+1}^{\prime}$, respectively, so none of the above edges exists. Furthermore, $x_{i+1} \neq x_{i+1}^{\prime}$ to avoid a claw at $x_{i+1}$.

But this implies that

$$
\left\langle v_{i+2} x_{i+2} x_{i} ; x_{i+1}^{\prime} x_{i+3} v_{i+4} P v_{i+k+2} ; x_{i+1} x_{i-1} v_{i-1} P v_{i-k+1}\right\rangle
$$




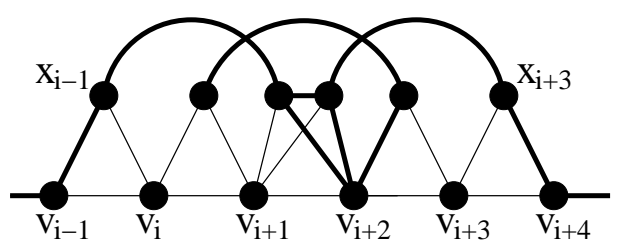

Figure 6. The configuration in the proof of Claim 4

is a copy of $F$, a contradiction.

The last two claims guarantee that there are $s, t$ with $2 k+5 \leq s \leq$ $2 k+7$ and $N-2 k-7 \leq t \leq N-2 k-5$ such that $X_{L}=N\left[v_{s}\right] \cap N\left[v_{s+1}\right]$ and $X_{R}=N\left[v_{t-1}\right] \cap N\left[v_{t}\right]$ are cut sets of $G$. Let $L$ be the component of $G-X_{L}$ containing $v_{0}$, let $R$ be the component of $G-X_{R}$ containing $v_{N}$, and let $M=G-(L \cup R)$.

Observe that $\left\langle X_{L}\right\rangle$ is almost complete. The only edges which may be missing are pairs $x y$ with $x \in N\left(v_{s-1}\right) \backslash\left\{v_{s}\right\}$ and $y \in N\left(v_{s+2}\right) \backslash\left\{v_{s+1}\right\}$ as all other missing edges would lead to claws. The symmetric statement is true for $\left\langle X_{R}\right\rangle$.

Claim 5. The graph $M$ is pancyclic.

Let $Q$ and $S$ be two disjoint paths in $M$ from $X_{L}$ to $X_{R}$ such that $|V(Q \cup S)|$ is minimal, let $q_{L}, s_{L}, q_{R}, s_{R}$ be the end vertices of the paths. If $V(Q \cup S) \cap V(P)=\emptyset$, we replace $Q$ by $v_{s+1} P v_{t-1}$, not changing $|V(Q \cup S)|$. Otherwise, let $q \in V(Q \cup S) \cap V(P)$ such that $V(Q \cup$ $S) \cap V\left(v_{s+1} P q^{-}\right)=\emptyset$. By symmetry we may assume that $q \in V(Q)$. Then $\left|V\left(v_{s} q_{L} Q q\right)\right| \geq\left|V\left(v_{s} P q\right)\right|$, and we may replace $q_{L} Q q$ by $v_{s+1} P q$. Therefore, we may assume that $q_{L}=v_{s+1}$ and that $v_{t-1} \in\left\{q_{R}, s_{R}\right\}$.

Since $v_{s}$ has no neighbors in $V(M) \backslash X_{L}$ and $v_{t}$ has no neighbors in $V(M) \backslash X_{R}$ the paths $Q^{\prime}=v_{0} P v_{s+1} Q q_{R}\left(v_{t}\right) v_{t+1} P v_{N}$ and $S^{\prime}=$ $v_{0} P v_{s-1}\left(v_{s}\right) s_{L} S s_{R}\left(v_{t}\right) v_{t+1} P v_{N}$ are induced. Furthermore, $v_{s} q_{L} Q q_{R} v_{t}$ is distance preserving in $M-S$, and $v_{s} s_{L} S s_{R} v_{t}$ is distance preserving in $M-Q$.

Let $C$ be the cycle $v_{s} q_{L} Q q_{R} v_{t} s_{R} S s_{L} v_{s}$. Cycles of all lengths $\ell$ for $3 \leq \ell \leq|V(C)|$ can be found again in the same fashion as in the proof of Lemma 10. Observe also that all vertices in $N(C) \cap V(M)$ can be added to $C$ one-by-one by Lemma 6 . Note that this includes all vertices in $X_{L} \subset N\left[v_{s+1}\right]$ and $X_{R} \subset N\left[v_{t-1}\right]$, which can be inserted between $x_{s}$ and $x_{s+1}$ and between $x_{t-1}$ and $x_{t}$, respectively.

All vertices in $V(M) \backslash N[C]$ must be in $N\left(N\left[Q^{\prime}\right]\right) \cap N\left(N\left[S^{\prime}\right]\right)$ by Lemma 7 applied to $Q^{\prime}$ and $S^{\prime}$, and therefore any such vertex has at least three neighbors in $N\left(Q^{\prime}\right) \cap N\left(S^{\prime}\right)$. None of these neighbors is in 
$V(L \cup R)$, so in the same fashion as in Lemma 11, we can add these vertices one-by-one to $C$.

Now let $C$ be an $\ell$-cycle with $\ell>|V(M)|$. For the sake of contradiction assume that $G$ contains no $(\ell-1)$-cycle. Consider $C \cap L$. If $C$ contains a vertex outside $L$, this is a (possibly empty) collection of paths $P_{0}, P_{1}, \ldots$ Let $p_{i}, s_{i} \in V(C) \cap X_{L}$ be the predecessors and successors of the $P_{i}$ on $C$.

Claim 6. There is a path $P_{L}$ beginning and ending in $X_{L}$ with $V\left(P_{L}\right) \subseteq$ $V(C) \cap N[L]$ which contains all $P_{i}$ in any given order and direction.

Let $P_{i}, P_{j} \subset C$ as above. Let $y_{i}$ be the final vertex of $P_{i}$ and $x_{j}$ the first vertex of $P_{j}$. If $s_{i}=p_{j}$, then $P_{i}$ and $P_{j}$ are consecutive on $C$ and we can either skip $s_{i}$ in $C$ to get an $(\ell-1)$-cycle, or $\left\langle s_{i}, v_{s+1}, x_{j}, y_{i}\right\rangle$ is a claw, which is a contradiction.

If $s_{i} p_{j} \notin E$, then $s_{i} \in N\left(v_{s-1}\right)$ and $p_{j} \in N\left(v_{s+2}\right)$ (or the other way around, but we may assume the former). To avoid a claw centered at $p_{j}$, we have $v_{s} x_{j} \in E$, as well as $v_{s-1} x_{j} \in E$ to avoid a claw at $v_{s}$. Now $v_{s-2} x_{j} \notin E$ to avoid a claw at $x_{j}$, concluding that $s_{i} x_{j} \in E$ to avoid a claw at $v_{s-1}$. Thus, we can connect $P_{i}$ and $P_{j}$ through $s_{i}$.

Similarly as $P_{L}$, we can construct a (possibly empty) path $P_{R}$ in $N[R]$. Next, we want to use paths similar to $Q$ and $S$ from above and join them with $P_{L}$ and $P_{R}$ into one big cycle $C^{\prime}$. We want to be able to add all remaining vertices in $M$ to $C^{\prime}$, so we have to take some care how we choose $P_{L}, P_{R}, Q$ and $S$.

If $v_{s}=p_{i}$ (or $v_{s}=s_{i}$ ) for some $P_{i} \subset P_{L}$, let $x_{L}=s_{i}$ (or $x_{L}=p_{i}$, respectively). Otherwise, let $x_{L}=v_{0}$. Similarly define $x_{R}$ in relation to $v_{t}$. Let $Q$ and $S$ be two disjoint paths in $M-\left\{x_{L}, x_{R}\right\}$ from $X_{L}-\left\{x_{L}\right\}$ to $X_{R}-\left\{x_{R}\right\}$ such that $|V(Q \cup S)|$ is minimal, $v_{s+1} \in V(Q)$ and $v_{t-1} \in V(Q \cup S)$. Such paths exist since $G$ has no cut set consisting of $x_{L}, x_{R}$ and a third vertex.

Let $q_{L}, q_{R}, s_{L}, s_{R}$ be defined as above. By our choice of $x_{L}$, we can order the $P_{i}$ on $P_{L}$ such that $P_{L}$ does not contain $s_{L}$ or $v_{s}$ as internal vertices. Similarly construct $P_{R}$. Now connect $P_{L}$ with $Q$ and $S$ as follows. If $s_{L}$ is an end vertex of $P_{L}$, then $P_{L}$ and $S$ are already connected. If $s_{L}$ is adjacent to an end vertex of $P_{L}$ which is not $v_{s}$, connect these two vertices. Otherwise, connect $s_{L}$ with $P_{L}$ through $v_{s}$. Finally, connect $q_{L}=v_{s+1}$ to the other end vertex of $P_{L}$ (through $v_{s}$ if $v_{s}$ is not already used). Similarly connect $P_{R}$ with $Q$ and $S$ to create the cycle $C^{\prime}$ with $V\left(C^{\prime}\right)=V\left(P_{L} \cup P_{R} \cup Q \cup S\right) \cup\left\{v_{s}, v_{t}\right\}$. If one of $P_{L}$ and $P_{R}$ is empty, use the vertex $v_{s}$ or $v_{t}$ instead of the path.

Case 1. Suppose that $\left|V\left(C^{\prime}\right)\right|<\ell$. 
We can add most vertices in $V(M) \backslash V\left(C^{\prime}\right)$ one-by-one in the same fashion as in Claim 5. The only vertices which may pose problems are the ones which were inserted somewhere between $q_{L}$ and $s_{L}$ or between $q_{R}$ and $s_{R}$.

For these vertices, note that every vertex in $X_{L} \backslash V\left(C^{\prime}\right)$ can be inserted between $v_{s}$ and $v_{s+1}$ after inserting $v_{s}$ between $v_{s+1}$ and $P_{L}$ if possible. This is impossible only in the case that $v_{s}$ and $s_{L}$ are adjacent on $C^{\prime}$. In this case, every vertex in $X_{L} \backslash V\left(C^{\prime}\right)$ can be inserted between $v_{s}$ and $s_{L}$ or between $v_{s+1}$ and $P_{L}$ as every vertex in $X_{L}$ is adjacent to at least one of any two non-adjacent vertices in $X_{L}$.

Finally, for vertices $u \in N\left(X_{L}\right) \cap N\left(N\left[Q^{\prime}\right]\right) \cap N\left(N\left[S^{\prime}\right]\right) \cap V(M)$, note that every neighbor $x \in N(u) \cap X_{L}$ is connected to all vertices in $X_{L}$ and has no neighbors in $L$ (otherwise there would be a claw). So these vertices can be inserted in the same fashion as in Claim 5 without interfering with $P_{L}$, and similarly vertices in $N\left(X_{R}\right) \cap N\left(N\left[Q^{\prime}\right]\right) \cap$ $N\left(N\left[S^{\prime}\right]\right) \cap V(M)$.

This way, we can extend $C^{\prime}$ to an $(\ell-1)$-cycle, which is a contradiction.

Case 2. Suppose that $\left|V\left(C^{\prime}\right)\right| \geq \ell$.

Observe that $\left|V(M) \backslash V\left(C^{\prime}\right)\right| \geq N-4 k-16$ as $G$ is 3-connected and $|V(Q \cup S)|$ is minimal. Thus, either $\left|V\left(C^{\prime}\right) \cap N[L]\right|>\frac{N}{2}-2 k-8$ or $\left|V\left(C^{\prime}\right) \cap N[R]\right|>\frac{N}{2}-2 k-8$. By symmetry we may assume that $\left|V\left(C^{\prime}\right) \cap N[L]\right|>\frac{N}{2}-2 k-8$. Note that $\left\langle N[L] \cup\left\{v_{s}\right\}\right\rangle$ has minimum degree at least three and diameter at most $4 k$, so we can shorten the path $\left\langle V\left(C^{\prime}\right) \cap\left(N[L] \cup\left\{v_{s}\right\}\right)\right\rangle_{C^{\prime}}$ one vertex at a time keeping the same end vertices by Lemma 4 .

After going through this procedure possibly for several times, we eventually arrive at a cycle of length $\ell-1$, which is a contradiction.

\section{REFERENCES}

1. B. Bollobás, "Random Graphs", Academic Press, London, 1985.

2. R. Diestel, "Graph Theory", Springer-Verlag, New York Graduate Texts in Mathematics, Volume 173 (1997).

3. R.J. Faudree, Z. Ryjáček, I. Schiermeyer, Forbidden subgraphs and cycle extendability, J. Combin. Math. Combin. Comput. 19 (1995), 109-128.

4. R.J. Faudree, R.J. Gould, Characterizing forbidden pairs for hamiltonian properties, Discrete Math. 173 (1997), 45-60.

5. R.J. Gould, T. Łuczak, F. Pfender, Pancyclicity of 3-connected graphs: pairs of forbidden subgraphs, J. Graph Theory 47 (2004) 183-202.

6. T. Luczak, F. Pfender, Claw-free 3-connected $P_{11}$-free Graphs are Hamiltonian, J. Graph Theory 47 (2004) 111-121. 
Department of Mathematics and Computer Science, Emory UniverSity, Atlanta, GA 30322, USA

E-mail address: <rg@mathcs.emory.edu>

Faculty of Mathematics and Computer Science, Adam Mickiewicz University, 60-769 Poznań, Poland

AND

Department of Mathematics and Computer Science, Emory UniverSity, ATLANTA, GA 30322, USA

E-mail address: <tomasz@amu.edu.pl>

Institut für Mathematik, Universität Rostock, 18051 Rostock, GerMANY

E-mail address: <florian.pfender@uni-rostock.de> 